\title{
Elimination of Contrast Agent Gadobutrol with Sustained Low Efficiency Daily Dialysis Compared to Intermittent Hemodialysis
}

\author{
Hanno Bunz ${ }^{a-c}$ Otto Tschritter ${ }^{d}$ Michael Haap ${ }^{\mathrm{e}}$ Reimer Riessen ${ }^{\mathrm{e}}$ \\ Nils Heyne ${ }^{a-c}$ Ferruh Artunc ${ }^{a-c}$ \\ ${ }^{a}$ Department of Internal Medicine, Division of Endocrinology, Diabetology, Vascular \\ Medicine, Nephrology and Clinical Chemistry, University Hospital Tübingen, Tübingen,

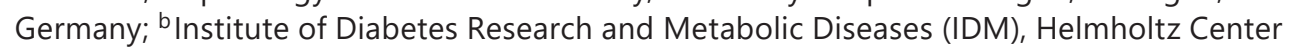 \\ Munich, University of Tübingen, Tübingen, Germany; ${ }^{\circ}$ German Center for Diabetes Research \\ (DZD), University of Tübingen, Tübingen, Germany; ${ }^{\mathrm{d}}$ Department of Emergency Medicine, \\ St. Mary's Hospital, Stuttgart, Germany; e Department of Internal Medicine, Internal \\ Intensive Care Unit, Tübingen, Germany
}

\section{Keywords}

Intermittent hemodialysis - Elimination of contrast agent gadobutrol ·

Sustained low efficiency daily dialysis

\begin{abstract}
Background: In patients with renal failure, gadolinium-based contrast agents (GBCA) can be removed by intermittent hemodialysis (iHD) to prevent possible toxic effects. There is no data on the efficacy of GBCA removal via sustained low efficiency daily dialysis (SLEDD) which is mainly used in intensive care unit (ICU) patients. Methods: We compared the elimination of the GBCA gadobutrol in 6 ICU patients treated with SLEDD (6-12 h, $90 \mathrm{~L}$ dialysate) with 7 normal ward inpatients treated with iHD (4 h, dialysate flow $500 \mathrm{~mL} / \mathrm{min}$ ). Both groups received 3 dialysis sessions on 3 consecutive days starting after the application of gadobutrol. Blood samples were drawn before and after each session and total dialysate, as well as urine was collected. Gadolinium (Gd) concentrations were measured using mass spectrometry and eliminated Gd was calculated from dialysate and urine. Results: The initial mean plasma Gd concentration was $385 \pm 183 \mu \mathrm{M}$ for the iHD and $270 \pm 97 \mu \mathrm{M}$ for the SLEDD group, respectively $(p>0.05)$. The Gd-reduction rate after the first dialysis session was $83 \pm 9$ and $67 \pm 9 \%$ for the iHD and the SLEDD groups, respectively $(p=0.0083)$. The Gd-reduction rate after the second and third dialysis was $94-98$ and $89-96 \%$ for the iHD and the SLEDD groups $(p>0.05)$. The total eliminated Gd was $89 \pm 14$ and $91 \pm 4 \%$ of the dose in the iHD and the SLEDD groups, respectively $(p>0.05)$. Gd dialyzer clearance was $95 \pm 22 \mathrm{~mL} / \mathrm{min}$ and $79 \pm 19 \mathrm{~mL} / \mathrm{min}$ for iHD and SLEDD, respectively $(p>0.05)$. Conclusions: Gd-elimination with SLEDD is equally effective as iHD and can be safely used to remove GBCA in ICU patients.




\section{Kidney \\ Blood Pressure \\ Research}

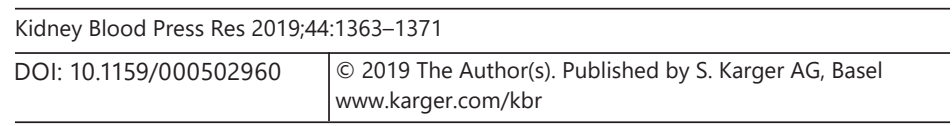

Bunz et al.: Elimination of Contrast Agent Gadobutrol with SLEDD Compared to iHD

\section{Introduction}

Gadolinium (Gd) belongs to the rare earths and although highly toxic to mammals it is used as a contrast agent in magnetic resonance imaging (MRI) due to its paramagnetic properties. To avoid acute toxicity, Gd is given as linear or cyclic chelates that are cleared mostly via glomerular filtration rate [1,2]. Gd release and its toxicity decreases with the stability of chelation, with higher stability in cyclic than in linear complexes [3, 4], which is explained by the mechanism of transmetalation $[5,6]$. Current studies have shown tissue deposition, especially in the brain even in patients with normal renal function [7-9]. These recent findings led to an EMA drug warning and prohibition of linear gadolinium-based contrast agents (GBCAs), however, clinical relevance is still lacking [10].

In contrast, a debilitating condition named nephrogenic systemic fibrosis (NSF) was first described between 2000 and 2001 [11,12] and related to Gd toxicity due to skin deposition in 2006 [13]. It was predominantly found in patients with severe renal failure including those with end-stage renal disease (ESRD). Up to now, the exact pathophysiology of NSF is unknown. It is suspected that free Gd may be released from the chelate and complexes with phosphate $[4,14]$. This hypothesis is supported by the detection of Gd in the tissue of NSF patients [15]. Incidence of NSF varies with the GBCA used, and in patients with chronic kidney disease it ranges from 0.0003 to $0.004 \%$, with higher rates in ESRD patients [16-19]. Among the various GDCA, gadodiamide has one of the lowest thermodynamic stability constants and one of the highest dissociation rates compared with other agents and has been the agent most commonly reported to be associated with the development of NSF [17]. To prevent possible toxicity from free $\mathrm{Gd}$, intermittent hemodialysis (iHD) has been shown to effectively remove Gd, reaching elimination rates of $>90 \%$ of the applied dose after 3 sessions [20-24]. In the study by Saitoh et al. [20], 74, 92, and 99\% of the gadodiamide dose was eliminated by the end of the first, second, and third session, respectively.

MRI is also an important imaging modality in intensive care unit (ICU) patients [25], who can be characterized by a high coincidence of acute kidney injury (AKI). From these, approximately 5\% need renal replacement therapy (RRT) using either intermittent or continuous hemodialysis modalities. Mortality for those in need of RRT reach $50-60$, and $13 \%$ of patients with RRT leaving ICU remain dialysis dependent [26-28]. Sustained low efficiency daily dialysis (SLEDD) is a hybrid hemodialysis modality in ICU patients, enabling daily hemodialysis sessions over a prolonged time. Its advantages include reduced nursing time and lower costs compared to CVVH at similar outcomes [29].

Because of significant efforts involved in the transportation of ICU patients, MRI seems to be underutilized and in ICU patients with AKI, application of GBCA might be another obstacle to MRI [30, 31]. Removal via hemodialysis in this population is not well known and it remains unclear if continuous or SLEDD might be equally effective as iHD, which is often poorly tolerated in ICU patients.

In this study, we investigated the elimination of the GBCA gadobutrol using SLEDD in ICU patients and compared the efficacy in ESRD patients using iHD. We demonstrated that Gd-elimination with SLEDD is equally effective as with iHD and can be safely used to remove GBCA in ICU patients.

\section{Materials and Methods}

Patients and Dialysis

We enrolled patients in need of a GBCA-enhanced MRI and dialysis at our hospital between 2016 and 2018. Exclusion criteria were age $<18$ years, residual urine output 
$>1,000 \mathrm{~mL} / 24 \mathrm{~h}$, and participation in another trial. The study was approved by the Institutional Review Board (IRB) and Ethics Committee (No: 281/2016MPG23). Fully informed and signed consent was obtained from each patient or his caregiver. We enrolled a total of 13 patients: 6 ICU patients with indication for contrast-enhanced MR imaging and dialysisdependent AKI and 7 ESRD patients. ICU patients received a SLEDD with a FMC Genius 90 dialysis machine (Fresenius Medical Care, Bad Homburg, Germany) and a $1.4 \mathrm{~m}^{2}$ high-flux Helixone membrane (F60; Fresenius Medical Care, Bad Homburg, Germany). Dialysis time and blood flow were individually adapted to the patient's hemodynamic status and ranged between 100-200 mL/min and 6-12 h, respectively. During SLEDD, exactly $90 \mathrm{~L}$ of dialysate equal to the total tank volume was spent. ESRD patients received an iHD using the FMC 5008 coreDiax dialysis machine (Fresenius Medical Care, Bad Homburg, Germany), a $1.4 \mathrm{~m}^{2}$ high-flux Helixone membrane (F60; Fresenius Medical Care, Bad Homburg, Germany) with a fixed time $(4 \mathrm{~h})$, a fixed dialysate flow $(500 \mathrm{~mL} / \mathrm{min})$, and a blood flow between 250 and $300 \mathrm{~mL} / \mathrm{min}$ (iHD group). Dialysis sessions were performed on 3 consecutive days starting on the day of application of gadobutrol (Gadovist, Bayer, $1 \mathrm{mmol} / \mathrm{mL}$ ) at a dose of $0.1 \mathrm{mmol} / \mathrm{kg}$ body weight. For every patient, the total volume (mL) of injected GBCA was noted.

To measure the Gd concentration, $5 \mathrm{~mL}$ blood was stored before and after each dialysis. In the iHD group, spent dialysate was collected in a tank, weighed and one sample was drawn. In the SLEDD group, dialysate samples were drawn from the dialysate tank according to the manufacturer's requirements. If patients had residual renal function, urine was collected, measured and samples were stored.

\section{Measurement of Gd in Serum, Urine and Dialysate}

To remove proteins, $1 \mathrm{~mL}$ serum was mixed with $1 \mathrm{~mL}$ nitric acid (65\%). The precipitated serum samples were separated by ultrafiltration to obtain a supernatant. Depending on the expected concentration, the samples were further diluted 1:100-1:5,000 using 1\% nitric acid $+0.01 \%$ Triton-X $100+5 \mathrm{nmol} / \mathrm{L}$ terbium as internal standard. Gd concentration was measured by Bayer AG (Berlin) using inductively coupled plasma mass spectrometry (ICP-M, Agilent 7900). The limit of quantification of the MS method was $0.1 \mathrm{nmol} / \mathrm{L}$ for the Gd isotope 158. All diluted samples were at least 10 -fold above the limit of quantification.

\section{Calculations}

The Gd-reduction rate was calculated for each dialysis session using the formula: $\left(\mathrm{c}_{\mathrm{GD}}\right.$ before $-\mathrm{c}_{\mathrm{GD}}$ after $) / \mathrm{c}_{\mathrm{GD}}$ before ${ }^{*} 100 \%$, whereby $\mathrm{C}_{\mathrm{GD}}$ denotes the $\mathrm{Gd}$ concentration. Rebound was calculated from $\mathrm{c}_{\mathrm{GD}}$ before next dialysis $\mathrm{C}_{\mathrm{GD}}$ after previous dialysis $/ \mathrm{C}_{\mathrm{GD}}$ after previous dialysis ${ }^{*} 100 \%$.

The percentage of eliminated Gd was calculated as: $\mathrm{n}_{\mathrm{GD} \text { collected }} / \mathrm{n}_{\mathrm{GD} \text { injected }}{ }^{*} 100 \%$, whereby $\mathrm{n}_{\mathrm{GD}}$ denotes the amount of $\mathrm{Gd}$.

The estimation of $\mathrm{Gd}$ dialyzer clearance was calculated as follows: $\mathrm{CL}_{\mathrm{GD}}=\mathrm{N}_{\mathrm{GD}}$ collected $/$ $\mathrm{AUC}_{\mathrm{GD} \text {-concentration before and after } \mathrm{HD}}$. $\mathrm{AUC}$ was calculated using the triangle formula: $\left(\mathrm{C}_{\mathrm{GD} \text { before }} \mathrm{HD}^{+}\right.$ $\left.\mathrm{C}_{\mathrm{GD} \text { after HD}}\right) / 2{ }^{*} \mathrm{t}_{\text {dialysis }}\left(\mu \mathrm{mol}{ }^{*} \mathrm{~min} / \mathrm{mL}\right)$.

In addition, we normalized the post-dialysis Gd concentration to the change of volume status (VS) of the patient by changes in serum-albumin concentrations. The amount of albumin is not affected by dialysis $\left(\mathrm{n}_{\text {pre }}=\mathrm{n}_{\text {post }}\right)$. Pre-dialysis VS was defined as $1\left(\mathrm{~V}_{\text {pre }}=1\right)$. The VS postdialysis is affected by fluid intake or ultrafiltration during dialysis. The formula $n_{\text {pre }}=n_{\text {post }}$ equals $c_{\text {pre }} * V_{\text {pre }}=c_{\text {post }} * V_{\text {post }}$. Therefore, the relative change in VS is defined as $V_{\text {post }}=c_{\text {pre }} / c_{\text {post }}$.

We corrected the $\mathrm{c}_{\mathrm{GD}}$ after by multiplying with $\mathrm{V}_{\text {post }}$ and calculated $\mathrm{GdRR}$ and $\mathrm{CL}_{\mathrm{Gd}}$ also with these corrected values. The amount of serum of subject 3 was too small, resulting in a total number of $n=12$ (6 iHD; 6 SLEDD) for the calculations with corrected VS. 
Table 1. Characteristics of patients

\begin{tabular}{lcc}
\hline & iHD & SLEDD \\
\hline Age, years & $58 \pm 17$ & $60 \pm 13$ \\
Height, m & $1.71 \pm 0.09$ & $1.75 \pm 0.14$ \\
Weight, kg & $73.2 \pm 15.1$ & $85 \pm 29.8$ \\
BMI, kg/m ${ }^{2}$ & $25 \pm 3.2$ & $27.2 \pm 6.1$ \\
Female/male, \% & $43 / 57$ & $17 / 83$ \\
Urea distribution volume & & \\
$\quad$ (Watson formula), L & $38 \pm 8$ & $43.3 \pm 12.8$ \\
Hypertension, $n(\%)$ & $6 / 7(86)$ & $2 / 6(33)$ \\
Atrial fibrillation, $n(\%)$ & $1 / 7(14)$ & $2 / 6(33)$ \\
Renal disease, $n(\%)$ & $1 / 7(14)$ & $5 / 6(83)$ \\
$\quad$ AKI & $2 / 7(28)$ & $0 / 6(0)$ \\
$\quad$ SLE & $1 / 7(14)$ & $1 / 6(17)$ \\
$\quad$ HRS & $1 / 7(14)$ & $0 / 6(0)$ \\
$\quad$ Ntx failure & $1 / 7(14)$ & $0 / 6(0)$ \\
$\quad$ Nephrectomy & $1 / 7(14)$ & $0 / 6(0)$ \\
$\quad$ Toxic &
\end{tabular}

Baseline characteristics of the iHD group and the SLEDD group. BMI, body mass index; AKI, acute kidney injury; SLE, systemic lupus erythematosus; HRS, hepatorenal syndrome; Ntx, kidney transplantation; iHD, intermittent hemodialysis; SLEDD, sustained low-efficiency daily dialysis.

\section{Statistics}

Statistical analysis was performed using the SAS JMP program (SAS Institute, Cary, NC, USA). We calculated descriptive statistics using sample sizes, arithmetic means for gadobutrol concentrations, SD 95\% CI of the mean where appropriate. Groups were compared using the $t$ test. A $p$ value $<0.05$ was considered statistically significant.

\section{Results}

The subject characteristics of the patients in both groups are shown in Table 1 . The cause of acute renal failure from acute tubular necrosis with consecutive dialysis was found in 5 out of 6 patients in the SLEED group. One patient was diagnosed with hepatorenal syndrome. In the iHD group, chronic dialysis dependent kidney disease was due to systemic lupus $(2 / 7$; $28 \%)$, bilateral nephrectomy $(1 / 7 ; 14 \%)$, transplant failure $(1 / 7 ; 14 \%)$, ifosfamide toxicity $(1 / 7 ; 14 \%)$, hepatorenal syndrome $(1 / 7 ; 14 \%)$, and 1 case of status post-AKI $(1 / 7 ; 14 \%)$. The median residual urine output was $266 \mathrm{~mL} / 24 \mathrm{~h}(533 ; 0 \mathrm{~mL} / 24 \mathrm{~h})$ and $216 \mathrm{~mL} / 24 \mathrm{~h}(2,300 ; 0$ $\mathrm{mL} / 24 \mathrm{~h}$ ) for iHD and SLEDD groups ( $p>0.05$; Table 2).

The initial mean serum Gd concentration was $385 \pm 183 \mu \mathrm{mol} \mathrm{Gd} / \mathrm{L}$ and $271 \pm 97 \mu \mathrm{mol}$ Gd/L for the iHD and the SLEDD groups, respectively $(p>0.05)$. After 3 dialysis sessions, Gd-serum concentrations fell to $5 \pm 4 \mu \mathrm{mol} \mathrm{Gd} / \mathrm{L}$ in the iHD and to $10 \pm 8 \mu \mathrm{mol} \mathrm{Gd} / \mathrm{L}$ in the SLEDD group ( $p>0.05$; Fig. 1). The Gd-reduction rate for the first dialysis session was $83 \pm 9$ and $67 \pm 9 \%$ for the iHD and the SLEDD groups, respectively ( $p=0.0083$; Fig. 2). The Gd-reduction rate for the second and third dialysis sessions was $94 \pm 4$ and $98 \pm 1 \%$ in the iHD group and $89 \pm 5$ and $96 \pm 3 \%$ in the SLEDD group, respectively ( $p>0.05$; Fig. 2 ).

When corrected for VS changes during dialysis, the Gd-reduction rate for the first dialysis session was $82 \pm 7$ and $68 \pm 10 \%$ for the iHD and the SLEDD groups, respectively ( $p=0.0197)$. 
Table 2. Gd-Clearance ( $\left.\mathrm{Gd}_{\text {clear }}\right)$ of each patient, mean urine volume per $24 \mathrm{~h}$, total amount of creatinine, BUN, and Gd in urine

\begin{tabular}{|c|c|c|c|c|c|c|c|}
\hline Group & Patient & $\begin{array}{l}\mathrm{Gd}_{\text {clear }} \\
1 \text { st } \mathrm{HD}, \mathrm{mL} / \\
\min \end{array}$ & $\begin{array}{l}\text { Mean Urine } \\
\text { volume, } \\
\mathrm{mL} / 24 \mathrm{~h}\end{array}$ & $\begin{array}{l}\text { Total } \\
\text { amount } \\
\text { of U-Crea, mg }\end{array}$ & $\begin{array}{l}\text { Total amoun } \\
\text { of U-BUN, } \\
\text { mg }\end{array}$ & $\begin{array}{l}\text { Total amount } \\
\text { of U-Gd, } \\
\mu \mathrm{mol}\end{array}$ & $\begin{array}{l}\text { Percentage } \\
\text { of total Gd, } \\
\%\end{array}$ \\
\hline \multirow[t]{8}{*}{ iHD } & 2 & 94.9 & 0 & 0 & 0 & 0 & 0 \\
\hline & 3 & 119.2 & 567 & 56 & 649 & 649 & 4.6 \\
\hline & 5 & 91.9 & 267 & 123 & 757 & 153 & 2.2 \\
\hline & 6 & 129.9 & 0 & 0 & 0 & 0 & 0 \\
\hline & 10 & 79.6 & 533 & - & - & 5 & 0.05 \\
\hline & 11 & 64.6 & 527 & 1,532 & 16,763 & 1,702 & 34.1 \\
\hline & 12 & 103.8 & 267 & 273 & 2,037 & 361 & 5.2 \\
\hline & Median & 94.9 & 267 & 89.5 & 703 & 153 & 2.2 \\
\hline \multirow[t]{7}{*}{ SLEDD } & 1 & 63.0 & 2,300 & 177 & 16,437 & 1,024 & 13.7 \\
\hline & 4 & 108.1 & 434 & 190 & 1,710 & 268 & 2.7 \\
\hline & 7 & 61.8 & 0 & 0 & 0 & 0 & 0 \\
\hline & 8 & 72.2 & 0 & 0 & 0 & 0 & 0 \\
\hline & 9 & 94.4 & 0 & 0 & 0 & 0 & 0 \\
\hline & 13 & 72.0 & 433 & 170 & 4,539 & 300 & 3.8 \\
\hline & Median & 72.1 & 216.5 & 85 & 855 & 134 & 1.35 \\
\hline
\end{tabular}

iHD, intermittent hemodialysis; SLEDD, sustained low efficiency daily dialysis.

Fig. 1. Mean serum Gd concentration before and after each dialysis session during iHD (blue line) and SLEDD (red line). iHD, intermittent hemodialysis; SLEDD, sustained low efficiency daily dialysis.

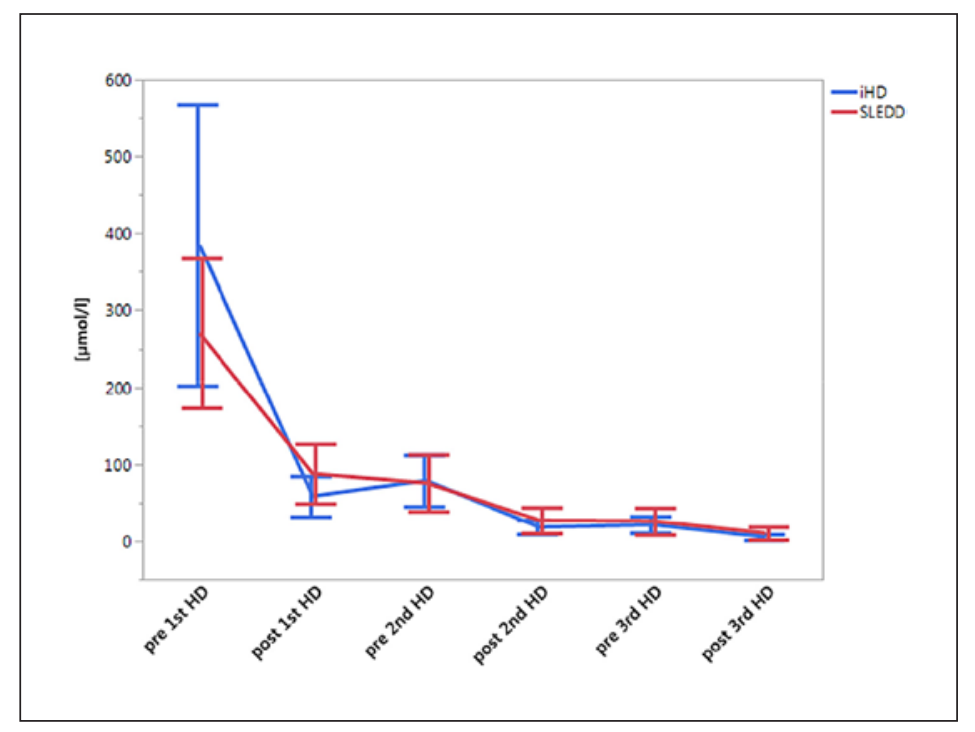

The Gd-reduction rate for the second and third dialysis sessions was $94 \pm 4$ and $98 \pm 1 \%$ in the iHD group and $90 \pm 5$ and $96 \pm 3 \%$ in the SLEDD group, respectively $(p>0.05)$.

Prior to the second dialysis session, there was a rebound of Gd-plasma concentration in the iHD group $(+22 \pm 25 \%)$ which was absent in the SLEDD group $(-18 \pm 12 \%, p=$ 0.0052 ; Fig. 3a). The rebound was reduced prior to the third dialysis session in the iHD group (13 $\pm 22 \%)$ and statistically not different from that seen in the SLEDD group $(-3 \pm$ $25 \% ; p>0.05)$. When corrected for VS changes, the rebound of Gd-plasma concentration in the iHD group persisted $(+16 \pm 19 \%)$ and was also absent in the SLEDD group $(-16 \pm$ $27 \% ; p=0.0384$ ). Again the rebound was reduced prior to the third dialysis session in 
Fig. 2. Gd reduction rates during the 3 consecutive dialysis sessions in patients treated with SLEDD or iHD. * Significant difference between the groups $(p<$ $0.05)$. iHD, intermittent hemodialysis; SLEDD, sustained low efficiency daily dialysis.
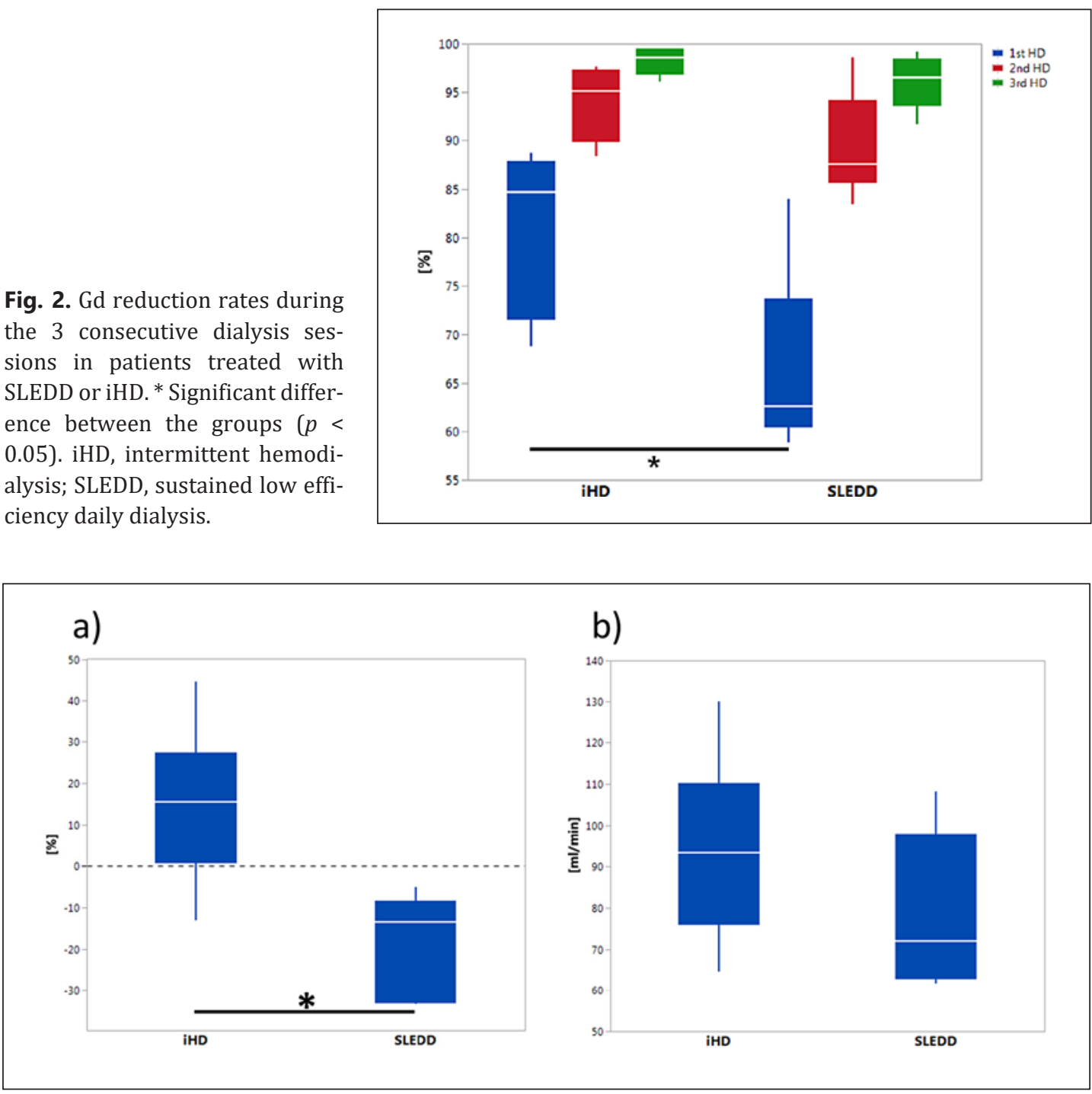

Fig. 3. Gd rebound rate between first and second dialysis sessions (a) and Gd clearance rate during first dialysis session in $\mathrm{mL} / \mathrm{min}(\mathbf{b}) .{ }^{*}$ Significant difference between the groups $(p<0.05)$. iHD, intermittent hemodialysis; SLEDD, sustained low efficiency daily dialysis.

the iHD group $(13 \pm 27 \%)$ and statistically not different from that seen in the SLEDD group $(4 \pm 32 \% ; p>0.05)$.

The mean dialyzer Gd clearance was 95 and $77 \mathrm{~mL} / \mathrm{min}$ for iHD and SLEDD groups, respectively ( $p>0.05$; Fig. 3b; Table 2$)$.

The total amount of eliminated Gd in all 3 dialysis sessions was $89 \pm 14 \%$ of the applied dose in the iHD group and $91 \pm 4 \%$ in the SLEDD group, respectively $(p>0.05)$. The percentage of eliminated Gd with urine was $7 \pm 12$ and $3 \pm 5 \%$ in the iHD and SLEDD groups, respectively $(p>0.05)$.

After 30 days of follow-up, 4 out of 6 patients in the SLEDD group had died and all patients in the iHD group were alive. There was no case or suspicion of NSF in any patient. One patient in the iHD group died during the next 12 months due to pneumonia. After a follow-up of 12 months, none of the patients developed NSF or another adverse advent that could be related to Gd toxicity. 


\section{Kidney \\ Blood Pressure \\ Research}

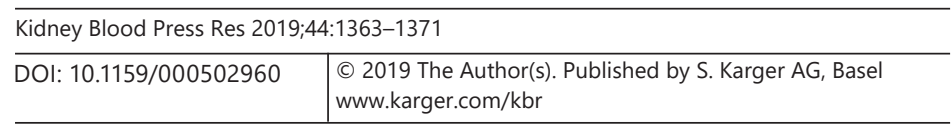

Bunz et al.: Elimination of Contrast Agent Gadobutrol with SLEDD Compared to iHD

\section{Discussion/Conclusion}

Our study is the first to analyze the elimination of a cyclic GBCA by SLEDD in ICU patients in comparison with iHD. Our results indicate that SLEDD eliminates gadobutrol with similar efficacy as iHD and achieved elimination rates of more than $90 \%$ of the administered Gd dose after 3 consecutive dialysis sessions. The remaining amount was excreted by residual kidney function. These data indicate that SLEDD is an adequate modality to eliminate GBCA in ICU patients who are usually hemodynamically compromised and poorly tolerate iHD. In these patients, dialysis modalities with a low blood flow such as SLEDD or continuous RRT are associated with improved hemodynamic stability. For continuous RRT, it is noteworthy that there are still no studies investigating the efficacy of Gd elimination [32]. Another advantage of SLEDD in the ICU over iHD is that it is more costeffective and does not require dialysis personnel during treatment since SLEDD is usually monitored by the ICU staff.

The plasma gadobutrol reduction rates achieved with iHD were higher than that reported by Tombach et al. [21], who reported values of $68 \%$ using a low-flux membrane with $1.2 \mathrm{~m}^{2}$. The difference can be explained by the longer dialysis time in our study. The Gd-RR of iHD was also higher compared to SLEDD, particularly in the first session indicating a more rapid clearance of Gd from the plasma compartment. This can be explained by the higher blood and dialysate flow during iHD. However, this was followed by a higher rebound as a result of Gd redistribution from the interstitial space. In an earlier study, we observed the same during dialysis with the drug dabigatran, which had higher reduction rate and rebound in iHD compared to SLEDD [33]. However, when looking at the eliminated Gd amount during the first session, there was no difference between SLEDD and iHD. These findings indicate that SLEDD eliminates Gd equally effectively due to the prolonged dialysis time that offsets the reduced blood and dialysate flow. It also shows that assessment of dialysis efficacy should not solely rely on plasma reduction rates that can overestimate clearance, especially when tissue deposition may occur $[7,9]$. Calculating dialyzer clearance from blood and dialysate samples also overestimate the true clearance [34]. The best way to assess the efficacy of any dialysis modality is the analysis of the spent dialysate as was done in this study. However, for the latter, sensitive quantification methods must be available given the large dialysate volume in which the analyte is diluted. So further studies on Gd elimination should take into account that a simple reduction rate is easy to use but is prone to overestimate the real clearance.

In conclusion, our study demonstrates that SLEDD is an adequate modality to effectively remove Gd in ICU patients with dialysis-dependent AKI and can be used to eliminate GDCA in ICU patients after Gd-enhanced MRI.

\section{Acknowledgement}

We thank Thomas Frenzel and the Bayer AG, Drug Discovery, Pharmaceuticals MR\&CT Contrast Media Research team for measuring the Gd concentrations. We acknowledge support by Deutsche Forschungsgemeinschaft and Open Access Publishing Fund of University of Tübingen. The contribution of Sebastian Hoerber is acknowledged.

\section{Statement of Ethics}

The study was approved by the IRB and Ethics Committee (No: 281/2016MPG23). Fully informed and signed consent was obtained from each patient or his caregiver. 


\section{Kidney \\ Blood Pressure Research}

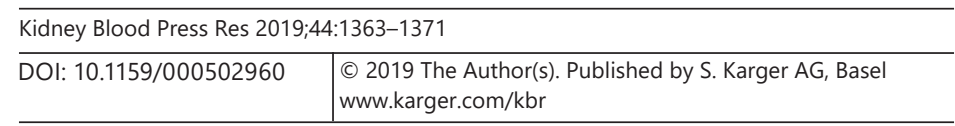

Bunz et al.: Elimination of Contrast Agent Gadobutrol with SLEDD Compared to iHD

\section{Disclosure Statement}

The authors have no conflicts of interest to declare.

\section{Funding Sources}

The study was carried out with own funds.

\section{Author Contributions}

H.B.: designed the study, was responsible for IRB approval, supervised the study and wrote main parts of the manuscript. N.H.: was involved in study design and manuscript revision. M.H. and R.R.: was involved in patient inclusion and data sampling. O.T.: was involved in study design pre-analytics and ethical approval. F.A.: was involved in study design, supervision, statistical analysis, and manuscript preparation.

\section{References}

1 Boss A, Martirosian P, Gehrmann M, Artunc F, Risler T, Oesingmann N, et al. Quantitative assessment of glomerular filtration rate with MR gadolinium slope clearance measurements: a phase I trial. Radiology. 2007 Mar;242(3):783-90.

2 Artunc F, Yildiz S, Boss A, Frenzel T, Schlemmer HP, Schick F, et al. Measurement of glomerular filtration rate using dynamic magnetic resonance imaging in patients with chronic kidney disease. J Nephrol. 2011 Jul-Aug; 24(4):482-9.

3 Mann JS. Stability of gadolinium complexes in vitro and in vivo. J Comput Assist Tomogr. 1993;17 Suppl 1:S1923.

4 Frenzel T, Lengsfeld P, Schirmer H, Hütter J, Weinmann HJ. Stability of gadolinium-based magnetic resonance imaging contrast agents in human serum at $37^{\circ}$ C. Invest Radiol. 2008 Dec;43(12):817-28.

5 Bousquet JC, Saini S, Stark DD, Hahn PF, Nigam M, Wittenberg J, et al. Gd-DOTA: characterization of a new paramagnetic complex. Radiology. 1988 Mar;166(3):693-8.

6 Idée JM, Port M, Raynal I, Schaefer M, Le Greneur S, Corot C. Clinical and biological consequences of transmetallation induced by contrast agents for magnetic resonance imaging: a review. Fundam Clin Pharmacol. 2006 Dec;20(6):563-76.

7 McDonald RJ, McDonald JS, Kallmes DF, Jentoft ME, Murray DL, Thielen KR, et al. Intracranial Gadolinium Deposition after Contrast-enhanced MR Imaging. Radiology. 2015 Jun;275(3):772-82.

8 Kanal E, Tweedle MF. Residual or retained gadolinium: practical implications for radiologists and our patients. Radiology. 2015 Jun;275(3):630-4.

9 Fitzgerald RT, Agarwal V, Hoang JK, Gaillard F, Dixon A, Kanal E. The Impact of Gadolinium Deposition on Radiology Practice: An International Survey of Radiologists. Curr Probl Diagn Radiol. 2019 May - Jun;48(3):22023.

10 EMA's final opinion on use of linear gadolinium agents. European Medicines Agency - Commission (2018). [accessed February 11, 2019] Available from: https://www.ema.europa.eu/en/medicines/human/referrals/ gadolinium-containing-contrast-agents.

11 Cowper SE, Robin HS, Steinberg SM, Su LD, Gupta S, LeBoit PE. Scleromyxoedema-like cutaneous diseases in renal-dialysis patients. Lancet. 2000 Sep;356(9234):1000-1.

12 Cowper SE, Su LD, Bhawan J, Robin HS, LeBoit PE. Nephrogenic fibrosing dermopathy. Am J Dermatopathol. 2001 Oct;23(5):383-93.

13 Grobner T. Gadolinium-a specific trigger for the development of nephrogenic fibrosing dermopathy and nephrogenic systemic fibrosis? Nephrol Dial Transplant. 2006 Apr;21(4):1104-8.

14 Zou Z, Zhang HL, Roditi GH, Leiner T, Kucharczyk W, Prince MR. Nephrogenic systemic fibrosis: review of 370 biopsy-confirmed cases. JACC Cardiovasc Imaging. 2011 Nov;4(11):1206-16.

15 High WA, Ayers RA, Chandler J, Zito G, Cowper SE. Gadolinium is detectable within the tissue of patients with nephrogenic systemic fibrosis. J Am Acad Dermatol. 2007 Jan;56(1):21-6.

16 Wang Y, Alkasab TK, Narin O, Nazarian RM, Kaewlai R, Kay J, et al. Incidence of nephrogenic systemic fibrosis after adoption of restrictive gadolinium-based contrast agent guidelines. Radiology. 2011 Jul;260(1):10511. 
17 Altun E, Martin DR, Wertman R, Lugo-Somolinos A, Fuller ER 3rd, Semelka RC. Nephrogenic systemic fibrosis: change in incidence following a switch in gadolinium agents and adoption of a gadolinium policy-report from two U.S. universities. Radiology. 2009 Dec;253(3):689-96.

18 Becker S, Walter S, Witzke O, Kreuter A, Kribben A, Mitchell A. Application of gadolinium-based contrast agents and prevalence of nephrogenic systemic fibrosis in a cohort of end-stage renal disease patients on hemodialysis. Nephron Clin Pract. 2012;121(1-2):c91-4.

19 Artunc F, Schanz S, Metze D, Heyne N. Nephrogene systemische Fibrose. Dtsch Med Wochenschr. 2008;133(S 00 Suppl):F1-1.

20 Saitoh T, Hayasaka K, Tanaka Y, Kuno T, Nagura Y. Dialyzability of gadodiamide in hemodialysis patients. Radiat Med. 2006 Jul;24(6):445-51.

21 Tombach B, Bremer C, Reimer P, Matzkies F, Schaefer RM, Ebert W, et al. Using highly concentrated gadobutrol as an MR contrast agent in patients also requiring hemodialysis: safety and dialysability. AJR Am J Roentgenol. 2002 Jan;178(1):105-9.

22 Okada S, Katagiri K, Kumazaki T, Yokoyama H. Safety of gadolinium contrast agent in hemodialysis patients. Acta Radiol. 2001 May;42(3):339-41.

23 Abe M, Kuno T, Takahashi Y, Nagura Y, Matsumoto K. [Evaluation of the elimination and safety of gadodiamide injection in patients with hemodialysis]. Nihon Jinzo Gakkai Shi. 2004;46(8):810-4.

24 Gheuens E, Daelemans R, Mesens S. Dialysability of gadoteric acid in patients with end-stage renal disease undergoing hemodialysis. Invest Radiol. 2014 Aug;49(8):505-8.

25 Barnett GH, Ropper AH, Johnson KA. Physiological support and monitoring of critically ill patients during magnetic resonance imaging. J Neurosurg. 1988 Feb;68(2):246-50.

26 Uchino S, Kellum JA, Bellomo R, Doig GS, Morimatsu H, Morgera S, et al.; Beginning and Ending Supportive Therapy for the Kidney (BEST Kidney) Investigators. Acute renal failure in critically ill patients: a multinational, multicenter study. JAMA. 2005 Aug;294(7):813-8.

27 Hoste EA, Schurgers M. Epidemiology of acute kidney injury: how big is the problem? Crit Care Med. 2008 Apr; 36(4 Suppl):S146-51.

28 Chawla LS, Eggers PW, Star RA, Kimmel PL. Acute kidney injury and chronic kidney disease as interconnected syndromes. N Engl J Med. 2014 Jul;371(1):58-66.

29 Schwenger V, Weigand MA, Hoffmann O, Dikow R, Kihm LP, Seckinger J, et al. Sustained low efficiency dialysis using a single-pass batch system in acute kidney injury - a randomized interventional trial: the REnal Replacement Therapy Study in Intensive Care Unit PatiEnts. Crit Care. 2012 Jul;16(4):R140.

30 Cournane S, Creagh D, O’Hare N, Sheehy N, Silke B. MRI in acutely ill medical patients in an Irish hospital: influence on outcomes and length of hospital stay. J Am Coll Radiol. 2014 Jul;11(7):698-702.

31 Kumarasamy N, Tishbi N, Mukundan S, Shiloh A, Levsky JM, Haramati LB. Cardiothoracic MRI in the ICU: A 10-Year Experience. Acad Radiol. 2018 Mar;25(3):359-64.

32 Schieda N, Blaichman JI, Costa AF, Glikstein R, Hurrell C, James M, et al. Gadolinium-Based Contrast Agents in Kidney Disease: A Comprehensive Review and Clinical Practice Guideline Issued by the Canadian Association of Radiologists. Can J Kidney Health Dis. 2018 Jun;5:2054358118778573.

33 Artunc F, Muehlbacher T, Baumann D, Birschmann I, Kuhn J, Heyne N, et al. Removal of Dabigatran Is Superior by Sustained Low Efficient Dialysis (SLED) Compared to Intermittent Hemodialysis. Blood Purif. 2015;39(4): $331-2$.

34 Tieu A, Velenosi TJ, Kucey AS, Weir MA, Urquhart BL. $\beta$-Blocker Dialyzability in Maintenance Hemodialysis Patients: A Randomized Clinical Trial. Clin J Am Soc Nephrol. 2018 Apr;13(4):604-11. 